\section{Medicinas Alternativas e Complementares: uma metassíntese}

\author{
Complementary and Alternative Medicines: \\ a meta-synthesis
}

1 Faculdade de Ciências Médicas, Universidade Estadual de Campinas, Campinas, Brasil.

2 Instituto de Saúde Coletiva

Universidade Federal da

Bahia, Salvador, Brasil.

${ }^{3}$ Healthcare School,

University of Leeds,

Leeds, U.K.

${ }^{4}$ Faculty of Health Sciences,

The University of Sydney,

Sydney, Australia.

Correspondência

C. Spadacio

Faculdade de Ciências

Médicas, Universidade

Estadual de Campinas.

Rua Tessália Vieira de

Camargo 126,

Campinas, $S P$

13083-790, Brasil.

cris.spadacio@gmail.com

Cristiane Spadacio 1

Marcelo Eduardo Pfeiffer Castellanos 2

Nelson Filice de Barros 1

Sarah Monte Alegre 1

Philip Tovey ${ }^{3}$

Alex Broom 4

\title{
Introdução
}

The growing use of Complementary and Alternative Medicines (CAM) has led to an increase in the number of qualitative studies on the subject, thus justifying a meta-synthesis of the resulting material. The current article presents a systematic review of qualitative studies on CAM published in international journals. The review was conducted according to the metasynthesis methodology. A search was performed in journals through the Periodicals Periodical of CAPES, the National Agency for the Evaluation of Graduate Studies, and 32 articles were selected for analysis. The reviewed studies raise questions focusing on: patients, their therapeutic experiences, and their social and cultural contexts; professionals, professional relations, and the process of professionalization of CAM; and CAM and their relationship to biomedicine. The article concludes that qualitative studies on CAM call for an exploratory view of the theme, seeking to identify the experiences of patients and professionals with these therapies and discussing the impact of their use on conventional medicine or biomedicine.

Qualitative Research; Complementary Therapies; Meta-Analysis
Tem havido um aumento exponencial no uso de Medicinas Alternativas e Complementares (MAC) no tratamento de várias doenças agudas e crônicas, dentre elas o câncer e o diabetes. O processo de ampliação no uso ocorre em paralelo ao progresso científico e tecnológico da medicina moderna ocidental e vem despertando o interesse de usuários, pesquisadores, profissionais e gestores de serviços de saúde.

A polêmica inserção das MAC nos sistemas de saúde nacionais tem como referência documentos da Organização Mundial da Saúde (OMS), como Estratégia de la OMS sobre Medicina Tradicional 2002-2005, preconizando investigações sobre: políticas de integração nacional dessas práticas nos sistemas nacionais de saúde; segurança, qualidade e eficácia dessas práticas; acesso às práticas e uso racional por profissionais e consumidores 1 .

Tal cenário tem estimulado a investigação de questões não exploradas nos estudos quantitativos, quase sempre empenhados em descrever: o tipo de técnicas utilizadas, o perfil da população consumidora e o tipo de evidência científica produzida sobre essas práticas 2. É perceptível o crescimento do número de estudos com abordagem qualitativa em saúde que buscam entender aspectos tais como as concepções pessoais e coletivas da doença, prevenção, risco, gerenciamento de condições crônicas, itinerários pelos 
serviços de saúde e escolhas terapêuticas. Além disso, identifica-se a necessidade de sistematizar o conhecimento científico que tem como referência a abordagem qualitativa sobre o tema das MAC, apontando as implicações desse conhecimento para o campo da Saúde.

O método utilizado para a produção de sínteses e evidências qualitativas é genericamente denominada "metassíntese" e permite realizar uma leitura crítica das interpretações presentes nos estudos qualitativos, norteando-se por parâmetros metodológicos rigorosos, sistemáticos e objetivos $3,4,5,6,7$.

Dentre as diferentes abordagens para a construção de metassínteses, o metaestudo 8 se destaca para análises de grande número de estudos e por estimular a cooperação e o trabalho conjunto entre pesquisadores. Na produção de metaestudos formulam-se, primeiramente, sínteses separadas a respeito dos aspectos teóricos, metodológicos e das análises de dados produzidas no conjunto de pesquisas revisadas, para então, num segundo momento, serem desenvolvidas as análises sintéticas sobre as implicações dos achados para o campo de conhecimento em que o tema é desenvolvido.

Este artigo apresenta um metaestudo de pesquisas qualitativas sobre MAC publicadas em periódicos indexados disponíveis no Portal Periódicos da Coordenação de Aperfeiçoamento de Pessoal de Nível Superior (CAPES; http://www. periodicos.capes.gov.br).

\section{Material e métodos}

Realizou-se a busca de artigos publicados em revistas presentes no Portal Periódicos. Esse portal disponibiliza a todos os programas de pós-graduação, reconhecidos por ela no Brasil, acesso integral e gratuito aos textos publicados em importantes revistas de circulação nacional e internacional. Ainda que o portal não inclua um universo de periódicos da área da saúde tão vasto quanto bases de dados internacionais consagradas (como MEDLINE e PubMed), ele apresenta a grande vantagem de superar essas bases no que diz respeito a textos integrais gratuitamente disponibilizados à comunidade científica brasileira. Além disso, ele opera em um universo de publicações internacionais em muito superior ao do SciELO, justificando-se assim a sua escolha. Por fim, o Portal Periódicos representa um esforço institucional valioso das políticas públicas voltadas à produção e circulação do conhecimento científico no Brasil, sendo objeto de constantes investimentos e aperfeiçoamentos, a fim de aumentar o seu acesso pela comunidade científica nacional. Torna-se, assim, relevante a realização de revisões sistemáticas nesse universo.

\section{Procedimentos de busca e seleção}

Inicialmente, foram selecionadas revistas relacionadas à: "social sciences", "qualitative resear$c h$ " e "complementary and alternative medicine" (CAM). Nessa primeira fase de seleção foram incluídos 27 periódicos.

Posteriormente, foram utilizados os descritores "CAM" e "qualitative" para o recrutamento final dos artigos a serem analisados nos 27 periódicos selecionados previamente. Mediante busca interna nesses periódicos, foram identificadas publicações sobre o tema nas seguintes revistas: Journal of Alternative and Complementary Medicine; Complementary Health Practice Review; Social Science \& Medicine; Evidence-Based Complementary and Alternative Medicine (eCAM) e Qualitative Health Research.

A seleção dos artigos foi realizada de forma independente por dois membros da equipe de pesquisa, pela leitura dos seus resumos. Em seguida, as discrepâncias na seleção foram discutidas e eliminadas com base na leitura dos artigos originais.

\section{Critérios de inclusão e de exclusão}

Foram incluídos os artigos originais referentes a pesquisas qualitativas que produziram dados primários sobre MAC, publicados no período de 1997-2008. Foram excluídos artigos que não atenderam aos critérios de inclusão. No total foram selecionados 32 artigos 9,10,11,12,13,14,15,16,17,18, $19,20,21,22,23,24,25,26,27,28,29,30,31,32,33,34,35,36,37,38,39,40$.

\section{Análise dos artigos}

Os artigos selecionados foram lidos na íntegra, fichados e classificados segundo sua temática principal. Essa classificação foi verificada e discutida pela equipe de pesquisadores.

Os fichamentos foram tabulados em uma planilha do Excel 2003 (Microsoft Corp., Estados Unidos), o que permitiu a sistematização das principais características dos estudos revisados. Para a tabulação das informações contidas nas pesquisas foram construídas quatro tabelas: a primeira, de identificação dos artigos selecionados contendo nome dos autores, título do artigo, referência, ano e país onde a pesquisa foi desenvolvida; a segunda, que congregou dados sobre a teoria; a terceira contendo os métodos utilizados nas pesquisas; e a quarta, referente à análise dos dados. 
Seguindo, dessa maneira, a proposta metodológica do metaestudo, privilegiou-se:

- Na metateoria, a análise sobre os problemas, conceitos e teorias que levaram os pesquisadores a explorar determinadas questões;

- No metamétodo, a análise da abordagem metodológica adotada nos estudos e de suas conseqüências para a perspectiva lançada sobre os problemas investigados;

- Na metanálise dos dados, ou seja, a análise das análises, confrontar as interpretações realizadas nos estudos revisados, reinterpretando os dados à luz dos achados dos demais estudos que compuseram o universo bibliográfico investigado.

Por intermédio dessas sínteses parciais, realizou-se uma síntese final, destacando as principais implicações das análises realizadas para o contexto científico, assistencial e das políticas públicas.

\section{Resultados}

\section{Análise metateórica}

Os estudos qualitativos analisados trazem questões que têm como focos de preocupação: o paciente, suas experiências terapêuticas e os contextos sociais e culturais que influenciam na decisão pelo uso ou não de MAC; o profissional, suas relações profissionais estabelecidas com base nas MAC e o processo de profissionalização de suas práticas como, por exemplo, a acupuntura; a MAC enquanto terapêutica que sofre um processo de legitimação atrelada à medicina convencional/biomedicina.

No que se refere às experiências de pacientes com a utilização de MAC, destaca-se a possibilidade de um cuidado integral focado na individualização do tratamento e os impactos positivos junto ao tratamento convencional. A comunicação do terapeuta de MAC e do profissional de saúde com o paciente mostrou-se, em alguns estudos, de extrema importância para as experiências terapêuticas dos pacientes, assim como a necessidade de levar em conta as condições sociais e as "barreiras" sócio-culturais para as escolhas terapêuticas 17,29 , aumentando, dessa maneira, a necessidade de mais pesquisas qualitativas para explorar as questões 31 .

Dentre os estudos que abordam a problemática das "relações profissionais", percebe-se a preocupação acerca dos poucos trabalhos que sistematizam as MAC e seu processo de profissionalização e institucionalização nos serviços de saúde. Incluem-se estudos que buscam compreender como se dá a prática terapêutica pela perspectiva dos próprios profissionais de MAC.
São realizados também questionamentos sobre como os profissionais de MAC se organizam para conseguir legitimidade no campo da Saúde, em busca da "oficialização" de suas práticas. Por se tratarem de pesquisas relacionadas à forma como se dão as relações profissionais no caso das MAC, a maioria dos estudos tem como foco a perspectiva dos profissionais.

Evidencia-se nesses estudos a preocupação com o aumento no uso de terapias não biomédicas, levando à necessidade de lidar com o atual pluralismo terapêutico, presente particularmente nas relações cotidianas 14 . Destaca-se que ainda se sabe pouco sobre a natureza do cuidado integral à saúde e a relação entre MAC e biomedicina no cotidiano dos serviços 19. Para Hsiao et al. 20, há evidências de que esses dois paradigmas não são combinados de maneira eficiente, especialmente pela falta de comunicação entre médicos e pacientes. Para isso, Barret et al. ${ }^{9}$ propõem a elaboração de novos modelos conceituais, de novas terminologias que definam com mais precisão o processo de integração entre MAC e medicina convencional (seguindo as indicações dos estudos analisados, tomamos como sinônimo os termos Medicina Convencional e Biomedicina, uma vez que os próprios artigos selecionados que não fazem essa distinção, referindo-se à biomedicina como equivalente da medicina convencional moderna).

Esses estudos estão mais preocupados com as formulações teóricas sobre a relação entre MAC e Medicina Convencional, sobretudo pela perspectiva dos profissionais de saúde ou da população de maneira geral, não exatamente de pacientes com alguma doença específica que se trate com MAC.

Existe um vasto repertório de conceitos e perspectivas teóricas utilizadas pelos trabalhos analisados: Grounded Theory 17,20,24,29,30,32,37,40, Fenomenologia 11,24, Hermenêutica 17,29, Boundary Theory ${ }^{36}$, teoria Stakeholder ${ }^{41}$, Closure Theory 19, a noção de Capital Cultural de Bourdieu 13; além de estudos que se pautam em referenciais da Sociologia da Saúde 31 e da Antropologia da Saúde 14 .

Um dos artigos analisados utiliza-se de uma combinação de abordagens, relacionando o modelo funcionalista, "social closure", sociologia das profissões e o conceito de compensação dos poderes 22. Ressalta-se que em alguns estudos as abordagens teóricas não foram relatadas $9,10,16,21,27,28,34,39$.

\section{Metamétodo}

De acordo com a perspectiva dos referenciais teóricos adotados pelos autores, os métodos de co- 
leta e análise dos dados utilizados pelas pesquisas também são variados. As entrevistas (semiestruturada ou em profundidade) 9,11,13,15,16,17,18, $19,20,21,22,23,24,25,26,27,28,29,30,31,32,33,34,35,36,37,38,39,40$ e os grupos focais 10,20,25,39 lideram as técnicas de coleta de dados utilizadas, seguidas por etnografia 10,13,14,19, análise documental 10,19 e histórias de vida escritas pelos próprios pacientes 35 .

Dos artigos selecionados, nove utilizam programas destinados à organização e à análise de dados qualitativos, com destaque para o NVivo (QRS International Pty, Doncaster, Austrália) e o Atlas.ti (Muhr T. Scientific software development GmbH, Berlim, Alemanha), seguidos pelo Ethnograph (Qualis Research Associates, Salt Lake City, Estados Unidos), pelo NUD.IST (QRS International Pty, Doncaster, Austrália) e pelo SAS versão 9.1 (SAS Inst., Cary, Estados Unidos).

A maior parte dos artigos, 21, não menciona a obtenção da aprovação da investigação em comitês de ética em pesquisa (CEP) e a utilização de termos de consentimento livre e esclarecido (TCLE) para a realização do trabalho de campo. Em relação ao restante, os autores não fazem qualquer reflexão mais aprofundada sobre os aspectos éticos envolvidos na pesquisa, geralmente, limitando-se a apontar os seus aspectos burocráticos - aprovação do CEP e utilização do TCLE - sem apontar ponderações sobre as implicações das estratégias metodológicas empregadas para os sujeitos investigados.

Poucos estudos declararam a técnica de análise de dados utilizada. Dentre as exceções, mencionou-se: análise de narrativa 18,34,36, modernidade reflexiva 38, Ottawa Decision Suport Framework (ODSF) 26, naturalistic inquiry methodology 33 , self-interview methodology 15 e phenomenographic methodology 35.

\section{Metanálise dos dados qualitativos}

A utilização das MAC encontra-se diretamente ligada ao alívio físico da dor, alívio emocional, diminuição dos efeitos colaterais de medicamentos alopáticos, melhora no funcionamento do sistema imunológico e aumento na qualidade de vida. Quanto ao processo de decisão pelo uso de MAC, evidenciam-se como fatores determinantes para o a utilização ou não dessas terapias: custo e capacidade de pagar pelas MAC; disponibilidade e acesso; tempo de tratamento com a biomedicina; rede de sociabilidade do paciente com o suporte familiar e de amigos; insatisfação com o tratamento médico convencional e busca por práticas que aliviem os sintomas das doenças.

No que se refere ao tema da profissionalização dos terapeutas de MAC e o oferecimento dessas práticas nos serviços de saúde, a biomedicina apresenta-se como termo de comparação ou parâmetro na definição de pressupostos de eficácia, requisitando a constante necessidade de comprovação científica. Para a institucionalização das MAC, Kelner et al. 22 acreditam ser necessário: melhorar a qualidade de programas educacionais sobre MAC, elevar a qualidade das práticas, desenvolver mais pesquisas sobre o tema e encontrar estratégias para deixar os vários grupos profissionais que compõem as MAC mais coesos.

Quanto às condições sociais e culturais para o uso ou não de MAC, Chacko 13 identifica correlações entre etnia e escolhas terapêuticas, enquanto outros autores Ohlen et al. 29 e Paterson et al. 31 chamam atenção para a necessidade de se criar um "lugar seguro", ou seja, um momento na interação clínica que propicie o diálogo entre médico e paciente, para que os pacientes possam tomar suas decisões quanto ao tratamento, respeitando a autonomia de escolha terapêutica. Podemos inferir, assim, que as preocupações sobre o uso de MAC e seu contexto social estão diretamente ligadas à figura do paciente e de suas escolhas terapêuticas.

Para Hollenberg 19, a postura da biomedicina e da MAC mostram-se semelhantes, sendo marcadas pela exclusão, dominação e demarcação. Enquanto a biomedicina usa os conhecimentos de MAC para dominá-la, a MAC se utiliza de tipos próprios de usurpação para resistir à biomedicina no interior dos serviços de saúde. O modelo integral de cuidado à saúde não existe nesse contexto, uma vez que os dois grupos se apropriam do conhecimento do outro para demarcar e reforçar seus próprios territórios.

Outra discussão evidenciada na literatura se refere às diferenças de racionalidades com as quais operam a biomedicina e as MAC em suas práticas terapêuticas. Nesse âmbito, descortinase a necessidade de novos modelos conceituais e terminologias que dêem conta de apreender o processo de integração entre biomedicina e MAC 9.

\section{Discussão: apontamentos para o campo da saúde}

A abordagem qualitativa em saúde nos permite apreender a complexa relação que se estabelece quando objeto, objetivos e formulações metodológicas são estruturados no processo criativo e dinâmico de pesquisa ${ }^{42}$. Este é um campo do conhecimento que se desenvolve por meio de práticas interpretativas por excelência. $\mathrm{O}$ qualitativo é eminentemente holístico e indutivo, toma co- 
mo referenciais o entendimento, a compreensão, a construção de sentido e a intencionalidade 43 .

Para o conjunto de artigos analisados, circunscritos no período de dez anos (1997-2008), as MAC são percebidas, de maneira geral, como parte de uma estratégia pragmática de manutenção da saúde, adotadas como forma de diminuir efeitos colaterais de tratamentos convencionais e como estratégia de aumentar a qualidade de vida. Além de representar uma escolha mais saudável quando comparada à medicina convencional.

Nessa direção, os trabalhos analisados têm como cerne de preocupação identificar os processos sociais que motivam e propiciam o uso de MAC pelos pacientes, os quais repercutem sobre suas experiências terapêuticas e/ou sobre as relações profissionais estabelecidas nesse campo de cuidados. Os arranjos teóricos e metodológicos delineados no enfrentamento dessa problemática, embora distintos, indicam um conjunto de reflexões de ordem microanalítica.

Tal perspectiva contribui para entendermos que compreender as MAC como um processo de escolha terapêutica socialmente construído em estreita relação com a Medicina Convencional auxilia profissionais de saúde e gestores a lidarem, no cotidiano institucional, com as diversas opções terapêuticas dos pacientes. Essa compreensão também permite aos pesquisadores o desenvolvimento de formulações conceituais e teóricas mais precisas, calcadas na perspectiva dos atores sociais envolvidos (pacientes e os profissionais de saúde).

É preciso, contudo, levar em consideração a necessidade de estudos de caráter qualitativo sobre o processo de formação de profissionais que atuem com essas Medicinas; sobre a incorporação, sistematização e financiamento das MAC nos sistemas nacionais de saúde; e até no que se refere à formulação de políticas públicas relativas ao tema. Essas questões se referem a um grau de amadurecimento e consolidação das MAC que já vem se mostrando presente em alguns contextos institucionais. No entanto, as reflexões sobre esse processo certamente requisitarão uma relação complementar entre as perspectivas micro e macro analíticas já que exige levar em consideração fatores estruturais, contextos institucionais específicos e interações entre profissionais e pacientes.

\section{Considerações finais}

Esse estudo tem o limite de ter analisado artigos de uma única fonte de dados bibliográficos, não contemplando a totalidade da produção acadêmico-científica sobre a abordagem qualitati- va das MAC. Ressalta-se, contudo, a relevância do Portal Periódicos da CAPES para a produção científica nacional porque nele se disponibilizam os trabalhos na íntegra, além da importância da construção de sínteses com dados de pesquisas qualitativas.

Com este trabalho foi possível identificar algumas características importantes no conjunto dos artigos analisados, evidenciando o estado da arte da temática das MAC, desenvolvidas mediante abordagem qualitativa. Destaca-se, em primeiro lugar, a variedade de objetos de estudos mapeados, a saber: aqueles que se referem às experiências terapêuticas dos pacientes e seus contextos sociais; aqueles que se preocupam em estudar como os profissionais de saúde lidam com as terapêuticas não biomédicas em sua prática profissional cotidiana; aqueles focados no processo de legalização e legitimação das MAC no interior da Medicina Convencional/Biomedicina. Essa multiplicidade de objetos estimula a existência de uma pluralidade de modelos teórico-metodológicos utilizados na investigação qualitativa das MAC.

Outro aspecto relevante abordado na literatura e que chama bastante atenção é a existência de uma discussão de cunho epistemológico. Por um lado, em relação ao fato de que a visibilidade das MAC é possível apenas em relação às práticas convencionais e, por outro, as notáveis diferenças nas lógicas de operação da medicina alopática, alicerçada no discurso sobre a cura, e das MAC, pautadas na manutenção da saúde.

Os estudos revisados nessa metassíntese sugerem a preocupação com a construção conceitual das MAC, que extrapola em muito a especulação teórico-diletante. Julga-se que a abordagem qualitativa é adequada para dar sustentação para essa construção, visto que examina os pontos de vista dos diferentes atores sociais, perguntandose pelo sentido e estruturação dessas práticas. Como conseqüência da perspectiva qualitativa, identificamos uma urgente necessidade de formulações teóricas e conceituais acerca das práticas terapêuticas cotidianas e as possíveis conseqüências para os serviços de saúde.

É perceptível, por fim, por intermédio das categorias identificadas nos estudos, a existência de um olhar ainda exploratório sobre diversas questões relativas ao tema das MAC, com ênfase na identificação das experiências dos pacientes e dos profissionais de saúde. Por isso, torna-se necessária a realização mais sistemática de estudos que busquem aprofundar a compreensão sobre os sentidos, entendimentos, percepções e experiências de pacientes e profissionais de saúde sobre o uso de práticas alternativas e complementares de cuidado e cura. 


\section{Resumo}

O aumento do uso de Medicinas Alternativas e Complementares (MAC) motivou o crescimento do número de estudos qualitativos sobre o tema, justificando a realização de sínteses sobre esse material. Este artigo apresenta uma revisão sistemática de pesquisas qualitativas sobre MAC publicadas em periódicos internacionais. Esta revisão se orientou pela metodologia dos metaestudos. Foi realizada busca em revistas do Portal Periódicos da Coordenação de Aperfeiçoamento de Pessoal de Nível Superior e foram selecionados 32 artigos para análise. Os estudos revisados apresentam questões que têm como foco: o paciente, suas experiências terapêuticas e seus contextos sociais e culturais; o profissional, as relações profissionais e o processo de profissionalização das MAC; a MAC e sua relação com a biomedicina. Conclui-se que as pesquisas qualitativas sobre as MAC ensejam um olhar exploratório sobre o tema, procurando identificar as experiências de pacientes e profissionais com essas terapêuticas, assim como buscam discutir as conseqüências desse uso para a Medicina Convencional ou biomedicina.

Pesquisa Qualitativa; Terapias Complementares; Metanálise

\section{Colaboradores}

C. Spadacio participou da redação do artigo. M. E. P. Castellanos e N. F. Barros aprovaram da versão final a ser publicada. S. M. Alegre, P. Tovey e A. Broom contribuíram na concepção e projeto, análise e interpretação dos dados.

\section{Referências}

1. Spadacio C, Barros NF. Uso de medicinas alternativas e complementares por pacientes com câncer: revisão sistemática. Rev Saúde Pública 2008; 42:158-64.

2. Tovey P, Chatwin J, Broom A. A traditional, complementary and alternative medicine and cancer care: an international analysis of grassroots integration. London: Routledge; 2007.

3. Thorne S, Jensen L, Kearney MH, Noblit G, Sandelowski M. Qualitative metasynthesis: reflections on methodological orientation and ideological agenda. Qual Health Res 2004; 14:1342-65.

4. Zhao S. Metatheory, metamethod, meta-dataanalysis: what, why, and how? Sociol Perspect 1991; 34:377-90.

5. Finfgeld DL. Metasynthesis: the state of the art - so far. Qual Health Res 2003; 13:893-904.

6. Sandelowski M, Barroso J. Writing the proposal for a qualitative research methodology project. Qual Health Res 2003; 13:781-820.
7. Bondas T, Hall EOC. Challenges in approaching metasynthesis research. Qual Health Res 2007; 17:113-21.

8. Thorne S, Paterson B, Acorn S, Canam C, Joachim G, Jillings C. Chronic illness experience: insights from a metastudy. Qual Health Res 2002; 12:437-52.

9. Barrett B, Marchand L, Scheder J, Plane MB, Maberry R, Appelbaum D, et al. Themes of holism, empowerment, access, and legitimacy define complementary, alternative and integrative medicine in relation to conventional biomedicine. J Altern Complement Med 2003; 9:937-47.

10. Boon H, Brown JB, Gavin A, Kennard MA, Stewart M. Breast cancer survivor's perceptions of complementary/alternative medicine (CAM): making the decision to use or not to use. Qual Health Res 1999; 9:639-53.

11. Cartwright T. Getting on with life: the experiences of older people using complementary health care. Soc Sci Med 2007; 64:1692-703. 
12. Cassiky CM. Chinese medicine users in the United States. Part II: preferred aspects of care. J Altern Complement Med 1998; 14:189-202.

13. Chacko E. Culture and therapy: complementary strategies for the treatment of type-2 diabetes in an urban setting in Kerala, India. Soc Sci Med 2003; 56:1087-98.

14. Connor LH. Relief, risk and renewal: mixed therapy regimens in an Australian suburb. Soc Sci Med 2004; 59:1695-705.

15. Elder WG, Purdy H, Bentley A. Collecting information about a CAM practitioner's practice: a preliminary report of a self-interview methodology. Complement Health Pract Rev 2005; 10:147-55.

16. Erickson BE. Radioactive pain relief: health care strategies and risk assessment among elderly persons with arthritis at radon health mines. J Altern Complement Med 2007; 13:375-9.

17. Foote-Ardah CE. Sociocultural barriers to the use of complementary and alternative medicine for HIV. Qual Health Res 2004; 14:593-611.

18. Hok J, Wachtler C, Falkengerg T, Tishelman C. Using narrative analysis to understand the combined use of complementary therapies and bio-medically oriented health care. Soc Sci Med 2007; 65:1642-53.

19. Hollenberg D. Uncharted ground: patterns of professional interaction among complementary/alternative and biomedical practitioners in integrative health care settings. Soc Sci Med 2006; 62:731-44.

20. Hsiao A, Ryan GW, Hays RD, Coulter ID, Andersen RM, Wenger NS. Variations in provider conceptions of integrative medicine. Soc Sci Med 2006; 62:2973-87.

21. Hussain-Gambles M, Tovey P. The experience of complementary alternative medicine among people with multiple sclerosis. Complement Health Pract Rev 2004; 9:21-30.

22. Kelner M, Wellman B, Welsh S, Boon H. How far can complementary and alternative medicine go? The case of chiropractic and homeopathy. Soc Sci Med 2006; 63:2617-27.

23. Kelner M, Wellman B, Boon H, Welsh S. Responses of established healthcare to the professionalization of complementary and alternative medicine in Ontario. Soc Sci Med 2004; 59:915-30.

24. Koithan M, Verhoef M, Bell IR, White M, Mulkins A, Ritenbaugh C. The process of whole person healing: unstuckness and beyond. J Altern Complement Med 2007; 13:659-68.

25. Laeeque H, Boon H, Kachan N, Cohen JC, D'Cruz J. The Canadian Natural Health Products (NHP) regulations: industry compliance motivations. Evid Based Complement Alternat Med 2007; 4:257-62.

26. Légaré F, Stacey D, Dodin S, O'Connor A, Richer M, Griffiths F, et al. Women's decision making about the use of natural health products at menopause: a needs assessment and patient decision aid. J Altern Complement Med 2007; 13:741-9.

27. MacPherson H, Thorpe L, Thomas K. Beyond needling - therapeutic processes in acupuncture care: a qualitative study nested within a low-back pain trial. J Altern Complement Med 2006; 12:873-80.
28. Magin PJ, Adams J, Heading GS, Pond DC, Smith W. Complementary and alternative medicine therapies in acne, psoriasis and atopic eczema: results of a qualitative study of patient's experiences and perceptions. J Altern Complement Med 2006; 12:451-7.

29. Ohlén J, Balneaves LG, Bottorff JL, Brazier ASA. The influence of significant others in complementary and alternative medicine decisions by cancer patients. Soc Sci Med 2003; 63:1625-36.

30. Paterson C, Britten N. Acupuncture as a complex intervention: a holistic model. J Altern Complement Med 2004; 10:791-801.

31. Paterson C, Zheng Z, Xue C, Wang Y. Playing their parts: the experience of participants in a randomized-sham-controlled acupuncture trial. J Altern Complement Med 2008; 14:199-208.

32. Pawluch D, Cain R, Gillet J. Lay constructions of HIV and complementary therapy use. Soc Sci Med 2000; 51:251-64.

33. Peck S. Aftermath of the unexpected, unexplained, and abrupt termination of health touch and extrapolation of related costs. Complement Health Pract Rev 2007; 12:140-60.

34. Prussing E, Sobo EJ, Walker E, Kurtin PS. Between desperation and disability rights: a narrative analysis of complementary/alternative medicine use by parents for children with Down syndrome. Soc Sci Med 2005; 60:587-9.

35. Ryan JD. Practices styles of beginner practitioners. J Altern Complement Med 2005; 11:477-82.

36. Shuval J. Nurses in alternative health care: integrating medical paradigms. Soc Sci Med 2003, 63:1784-95.

37. Shuval JT. Midwives practice CAM: feminism in the delivery room. Complement Health Pract Rev 2008; 13:46-62.

38. Tovey P, Broom A. Oncologist's and specialist cancer nurses' approaches to complementary and alternative medicine and their impact on patient action. Soc Sci Med 2007; 64:2550-64.

39. Verhoef MJ, Mulkins A, Boon H. Integrative health care: how can we determine whether patients benefit? J Altern Complement Med 2005; 11 Suppl 1:S57-65.

40. Warber SL, Cornelio D, Straughn J, Kile G. Biofield energy healing from the inside. J Altern Complement Med 2004; 10:1107-13.

41. Freeman RE. Strategic management: a stakeholder approach. Boston: Pitman Publishing; 1984. (Pitman Series in Business and Public Policy).

42. Bruyne P, Herman J, Schoutheete M. Dinâmica da pesquisa em Ciências Sociais: os pólos da prática metodológica. Rio de Janeiro: Francisco Alves; 1985.

43. Nunes ED. A metodologia qualitativa em saúde. Dilemas e desafios. In: Barros NF, Cecatti JG, Turato ER, organizadores. Pesquisa qualitativa em saúde: múltiplos olhares. Campinas: Universidade Estadual de Campinas; 2005. p. 15-24.

Recebido em 19/Jun/2009

Versão final reapresentada em 08/Out/2009

Aprovado em 10/Nov/2009 